\title{
Utilização de equipamento de proteção individual pela equipe de enfermagem em atenção paliativa em tempos de COVID-19
}

Use of Individual Protection Equipment by The Nursing Team in Palliative Attention in COVID-19 times

\section{Uso de Equipo de Protección Individual por el Equipo De Enfermería en Atención Paliativa en tiem- pos COVID-19}

\author{
Fernanda Barcellos Santiago ${ }^{1^{*}}$, Ana Lúcia Abrahão da Silva²
}

Como citar esse artigo. Santiago, FB; da Silva, ALA. Utilização de equipamento de proteção individual pela equipe de enfermagem em atenção paliativa em tempos de COVID-19. Revista Pró-UniverSUS. 2020 Jul./Dez.; 11 (2): 184-188.

\section{Resumo}

Objetivos: Analisar a segurança da equipe de enfermagem quanto a sua paramentação e desparamentação de EPIs frente a assistência de enfermagem a pacientes em tratamento paliativo oncológico acometidos pelo COVID -19 através da metodologia da simulação realística in situ. Método: Trata-se de Pesquisa Convergente-Assistencial (PCA) com abordagem qualitativa Resultados: As evidências geradas por esta pesquisa poderão embasar o uso da simulação realistica como estratégia de educação continuada para paramentação e desparamentação bem como lidar com EPIs contaminados para toda a equipe de enfermagem que presta assitência a pacientes suspeitos ou confirmados pelo COVID-19 em uma unidade de internação oncológica no Rio de Janeiro. Conclusão: Em um momento de pandemia, a importância da educação continuada é ressaltada para uma melhor organização e segurança dos profissionais de enfermagem.

Palavras-chave: Pandemia, Educação Continuada, Cuidados Paliativos, Equipamento de Proteção Individual.

\begin{abstract}
Objectives: To analyze the safety of the nursing team in terms of their attire and lack of PPE in relation to nursing care for patients undergoing palliative cancer treatment affected by COVID -19 through the methodology of realistic in situ simulation. Method: This is a Convergent-Assistance Research (PCA) with a qualitative approach Results: The evidence generated by this research may support the use of realistic simulation as a strategy of continuing education for paramentation and de-separation as well as dealing with contaminated PPE for the whole team nurse who assists patients suspected or confirmed by COVID-19 in an oncology inpatient unit in Rio de Janeiro. Conclusion: In a time of pandemic, the importance of continuing education is emphasized for better organization and safety for nursing professionals.

Keywords: Pandemic, Continuing Education, Palliative Care, Personal Protective Equipment.
\end{abstract}

\section{Resumen}

Objetivos: Analizar la seguridad del equipo de enfermería en términos de su vestimenta y la falta de EPP en relación con la atención de enfermería para pacientes sometidos a tratamiento de cáncer paliativo afectados por COVID -19 a través de la metodología de simulación realista in situ. Método: Esta es una Investigación de Asistencia Convergente (PCA) con un enfoque cualitativo. Resultados: La evidencia generada por esta investigación puede respaldar el uso de simulación realista como estrategia de educación continua para paramentación y des-separación, así como tratar con EPP contaminado para todo el equipo. Enfermera que ayuda a pacientes sospechosos o confirmados por COVID-19 en una unidad de hospitalización oncológica en Río de Janeiro. Conclusión: En tiempos de pandemia, se enfatiza la importancia de la educación continua para una mejor organización y seguridad para los profesionales de enfermería.

Palabras clave: Pandemia, Educación Continua, Cuidados Paliativos, Equipo de Protección Personal.

Afiliação dos autores:

${ }^{1}$ Enfermeira. Mestranda do Programa de Mestrado Profissional em Ensino na Saúde/MPES, UFF, RJ, Brasil. Email: nanda_barcellos@yahoo.com.br ORCID: https://orcid.org/00000001-7067-7234

${ }^{2}$ Enfermeira. Pós Doutora vinculada ao quadro Permanente do Programa de Ciências do Cuidado em Saúde e do Mestrado Profissional de Ensino em Saúde/MPES, UFF, Professora

Titular da Universidade Federal Fluminense, Escola de Enfermagem, RJ, Brasil. Email: abrahaoana@gmail.com ORCID: https://orcid.org/0000-0002-0820-4329 


\section{Introdução}

O cuidado paliativo é uma abordagem que tem como objetivo a promoção da qualidade de vida e de morte, em sua integralidade, e o alivio do sofrimento humano ${ }^{1}$.

Pacientes com câncer avançado em cuidados paliativos apresentam sobrevida reduzida ${ }^{2}$ e, em caso de infecção pelo COVID-19, experimentarão, provavelmente, uma redução ainda mais expressiva de vida ${ }^{3}$. A entubação, conduta usualmente adotada nos casos graves pelo COVID-19, medidas e manejos que não fazem parte do protocolo de assitência desses pacientes.

No contexto de uma doença altamente contagiosa como o COVID-19, faz sentido limitar ao mínimo os profissionais que tem contato direto com o paciente e estes profissionais devem possuir treinamento especifico para evitar a contaminação que além do adoecimento do Profissional acarretará inúmeros outros comprometimentos para o sistema de saúde, sobrecarga dos demais profissionais, colapso na assitência ao demais pacientes. $\mathrm{O}$ impacto de uma doença nova, o medo pessoal da sua própria contaminação e de ser possível veiculo de disseminação para a sua própria família, associado a escassez de EPI, faz com que as orientações e o treinamento sobre os cuidados, paramentação e desparamentação seja clara, continua e ágil pois pode ser um consolo ao Professional nesse momento de medos e incertezas ${ }^{4}$.

O processo educativo pode ser caracterizado como um cuidado da instituição para com os trabalhadores, no processo de trabalho.

Assim, podemos entender que a educação continuada, como um processo educativo dinâmico, dialógico, que visa a educação de determinado tema, no trabalho na área da saúde, estimulando a atuação crítica, reflexiva, buscando um melhor ambiente de trabalho, neste caso voltado para a construção de processo de trabalho ${ }^{5}$.

$\mathrm{Na}$ concepção de educação de Paulo freire, o homem é percebido como um ser autônomo, essa autonomia está presente na definição de "ser mais" que esta associada com a capacidade de transformar o mundo. A educação possibilita "aos oprimidos de libertarem-se da sua condição de oprimido, uma vez que, a liberdade é uma conquista e não doação, e exige uma permanente busca" ${ }^{\circ}$.

Assim, o ensino-aprendizagem no trabalho volta-se para a criação de um espaço transformador, em que os envolvidos possam refletir sobre a realidade do trabalho, conscientizar-se e buscar transformação. A partir de Paulo Freire os sujeitos aprendem a fazer essa ação libertadora através do processo de construção coletiva do saber, que revela os problemas existentes e instiga os próprios sujeitos na busca de soluções ${ }^{6}$.

Existem vários tipos de intervenções e práticas que podem ser utilizadas para colaborar com esse ensino - aprendizagem no âmbito de educação continuada. Porém a Pesquisa Convergente-Assistencial (PCA) foi escolhida por apresentar as questões de pesquisa emergindo da prática e o resultado destina-se 'a solução dos problemas e melhorar o ambiente do trabalho ${ }^{7}$.

A simulação realística in situ, poderá proporcionar uma melhor avaliação e orientação da conduta da equipe de enfermagem que cuida do paciente acometido pelo COVID-19 ou os casos suspeitos no que se refere a paramentação e desparamentação de todo o EPI necessário a proteção deste profissional que é a única categoria Profissional que permanece $24 \mathrm{~h}$ do dia nos cuidados ininterruptos ao paciente acometido ou suspeito do COVID-19. A mudança é possível a partir do momento que o profissional torna-se consciente da necessidade de mudar, porem para alcançar essa consciência, é preciso que haja dialogo, que pode ser proporcionado através da educação no trabalho. As mudanças acontecem quando percebemos que podemos fazer diferente e melhor.

Diante de tal problemática, a partir da experiência prática da autora, em um hospital especializado em cuidados paliativos oncológico, que presta cuidados de enfermagem a paciente com câncer em cuidados paliativos e acometidos pela COVID-19, e considerando que a equipe de enfermagem é a que mais se dedica aos pacientes e seus familiares no contexto da internação hospitalar, por permanecer 24 horas, incluindo período noturno.

Este estudo, busca utilizar o uso da simulação realística in situ no processo de paramentação e desparamentação bem como lidar com os EPIs contaminados após o cuidado de enfermagem a pacientes acometidos pelo COVID-19 e em cuidados paliativos oncológicos, associado a educação continuada da equipe de enfermagem que está diretamente envolvida na assistência destes usuários.

\section{Objetivo}

Analisar a segurança da equipe de enfermagem quanto a sua paramentação e desparamentação de EPIs frente a assistência de enfermagem a pacientes em tratamento paliativo oncológico acometidos pelo COVID -19 através da metodologia da simulação realística in situ.

\section{Metodologia}

Trata-se de uma pesquisa convergente assistencial (PCA), com abordagem qualitativa. O método de investigação PCA foi lançado em 1999 pelas 
enfermeiras e doutoras Mercedes Trentini e Lygia Paim, reconhecidas pela defesa da pesquisa como instrumento para promover mudanças no processo assistencial ${ }^{8}$.

Segue a lógica indutivo-dedutiva, em que o pesquisador assume o compromisso com a construção de um novo conhecimento, de novos modos de cuidado e de tecnologias, promovendo a renovação ou inovação da prática assistencial ${ }^{9}$.

Esta modalidade de pesquisa insere-se na pesquisa de campo no que se refere a prática assistencial pois articula a prática assistencial com conhecimento teórico e seus resultados são canalizados para situações práticas $^{10}$.

A PCA consiste em manter, durante o processo investigativo, uma estreita relação entre a pratica da pesquisa e a assistência.

A aplicação de estudos participativos em saúde do trabalho possibilita a construção de ações em estreita colaboração com os participantes o que potencializa os resultados. O processo de pesquisa participativa é por si só rico em gerar conteúdo e agilizar mudança entre os trabalhadores.

A PCA afasta a dicotomia entre a teoria e a prática. Ela possibilita refletir e aprimorar a prática profissional ${ }^{11}$.

Segundo Paim e Trentini, o PCA pretende unir método de pesquisa e método de prática assistencial e para a concepção do PCA, as seguintes fases devem ser construídas: concepção, instrumentação, perscrutação, analise e interpretação.

A fase de concepção consiste no problema de pesquisa, somente pode ser considerado um problema da PCA quando sua determinação for objeto com a equipe assistencial, que vai além do pesquisador.

Para tanto, a pesquisadora que também é enfermeira assistencial do setor de internação apresentou dificuldades em paramentar e se desparamentar ao terminar a assitência de enfermagem prestada a um paciente com diagnóstico de COVID -19. A partir deste fato, conversou com demais membros da equipe de enfermagem, técnicos e enfermeiros e relataram também ter dificuldades nesse contexto, bem como lidar com os EPI contaminados, pois cada um manipulava de um jeito e o acondicionava de forma própria. Então delimitou -se o tema do estudo (educação continuada através da simulação realistica para paramentação e desparamentação de EPIs na assitência de enfermagem a pacientes em cuidados paliativos oncológicos acometidos pelo COVID -19); definiu-se a questão norteadora( segurança da equipe de enfermagem na paramentação e desparamentação dos EPIs para a assistência de enfermagem ao paciente em cuidados paliativos oncológicos acometido pelo COVID-19) e estabeleceu como objetivo geral: (Analisar a segurança da equipe de enfermagem quanto a sua paramentação e desparamentação de EPIs frente a assistência de enfermagem a pacientes em tratamento paliativo oncológico acometidos pelo COVID -19 através da metodologia da simulação realística in situ )

A fase de instrumentação definiram-se o cenário de estudo (internação hospitalar do INCA IV) , os participantes (toda a equipe de enfermagem dos 4 andares de internação hospitalar), as técnicas de coleta de dados (observação participante e grupo focal que serão realizadas através da simulação realistica da paramentação e desparamentação dos EPI ,resposta ao check list e reunião após o termino do tipo grupo focal) e a análise qualitativa para cada técnica resultando na construção da avaliação final do treinamento.

A obtenção de dados e como não são estipuladas técnicas especificas, reúne diferentes métodos, desde que as informações obtidas na prática sejam reconhecidas como dados de pesquisa, utilizaremos a observação participante para coleta de dados ${ }^{12}$.

A fase de perscrutação, que significa investigar rigorosamente, embora descrita separadamente (didática) está relacionada a fase de instrumentação e análise. Inclui coleta e organização de resultados. Ao executar a pratica assistencial paralelamente observar os fatos investigados (pesquisa), o pesquisador registrará os acontecimentos de modo a dar continuidade a perscrutação até alcançar o conjunto de informações.

Foi elaborado um roteiro de observação para o treinamento das equipes onde a pesquisadora realizará os nomes dos participantes, local, hora e descrição do fato e do comportamento dos profissionais em realizar o treinamento.

A fase de interpretação: utilizaremos análise dos dados a estratégia serão 4 processos de análise qualitativa que ocorrerão simultaneamente à coleta de dados: apreensão, síntese, teorização e transferência ${ }^{12}$. Será realizada concomitante a produção dos dados.

\section{Resultados Esperados}

O impacto de uma doença com elevada taxa de contaminação e a possibilidade de desenvolvimento de casos graves pelo acometimento pulmonar severo e o fato de ser uma doença nova que traz incertezas em relação ao seu curso gera diversos sentimentos nos profissionais de saúde e principalmente nos da equipe de enfermagem por permanecerem de forma ininterrupta na assitência $24 \mathrm{~h}$ com pacientes acometidos ou suspeitos pelo COVID19. Esse medo pessoal, da sua própria contaminação e de ser um possível veiculo de contaminação para sua família em tempos de quarentena e reclusão para proteção das famílias, esta associado a escassez de EPIs e da falta de orientação clara dos serviços de educação continuada nos serviços hospitalares ${ }^{4}$.

Considera-se que o uso dos EPIs protege a integridade física do profissional, auxiliando na 
prevenção da exposição dos riscos biológicos a que a equipe de enfermagem está submetida. De acordo com a Norma Regulamentadora NR 06, considera-se EPI: todo dispositivo ou produto de uso individual do trabalhador, destinado à proteção de riscos capazes de ameaçar a segurança e a saúde no trabalho.

Nesse contexto, a simulação realística como processo de aprendizagem representa um potencial a ser estudado como um possível aliado, na prática profissional, quando nos deparamos com situações de pacientes em cuidados paliativos e acometidos pelo COVID-19.

A importância da atuação do enfermeiro paliativista é principalmente garantir a melhor qualidade de vida dos pacientes que estão sob seu cuidado. Sua necessidade de aprimoramento profissional é algo que faz parte da sua responsabilidade profissional, atuar de forma a reduzir as complicações do avançar da doença oncológica.

O profissional de saúde especializado em cuidados paliativos oncológicos possui atributos-chave para lidar com esta pandemia pois é responsável por aliviar sintomas, realiza tomada de decisões complexas e gerencia incertezas clinicas em seu dia a dia, logo possuem componentes essências em momentos de crise de saude publica como a que estamos vivendo na realidade da pandemia atual ${ }^{13}$.

Diante dessa situação crítica vivenciada em todo o mundo desde que a OMS decretou o COVID19 como uma nova pandemia, os profissionais de saúde que estão diretamente envolvidos no diagnóstico, tratamento e atendimento de pacientes com COVID19 correm o risco de desenvolver angústias e outros sintomas de saúde mental. O número sempre crescente de confirmados e casos suspeitos, acarreta a carga de trabalho maior, racionamento de EPIs, falta de medicamentos específicos, sentimento de falta de apoio das instituições de saúde, podem contribuir para a carga mental desses profissionais ${ }^{13}$.

Espera-se que com a execução plena desse projeto que está sendo proposto, seja possível acumular novos conhecimentos e consolidar anteriores, bem como subsidiar propostas de mudanças de rotina de paramentação e desparamentação bem como lidar com EPIs contaminados após a assitência de enfermagem a pacientes suspeitos ou casos confirmados pelo COVID19. Todos esses pontos são considerados importantes do ponto de vista da saúde pública.

Pretende-se preencher lacunas relacionados aos aspectos conflitantes na equipe de enfermagem proveniente do medo de contaminação. Os dados servirão para nortear e estimular a implementação do uso de simulação realística como estratégia de educação continuada para a equipe de enfermagem.

Ademais, procura-se contribuir para $\mathrm{O}$ enriquecimento da literatura cientifica com publicações desse tema, visando um impacto ainda mais amplo para o desenvolvimento da educação continuada no serviço hospitalar. A expectativa é que as evidências acumuladas possam trazer melhor compreensão e maior confiança do Professional de enfermagem que esta em cuidados diretos aos pacientes com suspeita ou confirmado para COVID-19.

\section{Considerações Finais}

Assim sendo, podemos afirmar que esse estudo possui relevância social, acadêmica e profissional, e que através da verificação do aumento da segurança do profissional, em estar paramentado de forma correta e de forma efetiva a se desparamentar evitando assim sua contaminação e também como lidar com todos os EPIs contaminados e sua desinfecção para novo uso, tornase crucial para sua auto confiança e poderá melhorar o estresse em lidar com essa nova doença. Bem como na assistência ao paciente.

\section{Referências}

1. Merino N, Reyes T, Reyes ME. Ética, Bioética y Legalidad en los uidados Paliativos; Competencia de Enfermería. Cancerología. 2010; 1(1): $37-44$

2. Silva, RCF, Hortale, VA. Cuidados paliativos oncológicos: elementos para debate de diretrizes nesta área. Cad. saúde pública. Rio de Janeiro, out 2006;22(10):2055 - 2066 .

3. Thueler, LCS , Melo, AC. Sars-CoV/COVID-19 em pacientes com câncer. Revista brasileira de cancerologia, 2020.

4. Verbeek JH et al. Personal protective equipment for preventing highly infectious diseases due to exposure to contaminated body fluids in healthcare staff. Cochrane Database of Systematic Reviews 2020;4. Available from: https://doi.org/10.1002/14651858.CD011621.pub4

5. Ricardone, CAC, Sena; RR. Educação Permanente: uma ferramenta para pensar e agir no trabalho de enfermagem. ver. Latino- Am. Enfermagem 2006;12(1): 80-88.

6. Freira, P. Pedagogia do oprimido. São Paulo: Paz e Terra . 1. ed, 1997

7. Pivoto FL, Lunardi Filho WD, Santos SSC, Lunardi VL. Convergentassistential research: an integrative review of scientific nursing production. Texto contexto - enferm. [Internet]. 2013 Sep [cited 2017 Jan 30]; 22(3): 843-849. Available from: http://www.scielo.br/scielo.php?script=sci arttext\&pid=S0104-07072013000300034\&lng=en. http://dx.doi. org/10.1590/S0104-07072013000300034 [ Links ]

8. Trentini, M. Paim, LE. Pesquisa convergente-assitencial e sua aplicação em cenários da enfermagem. Cogitare Enfermagem, 2008;13(3).

9. Rocha, PK, Prado, ML, Silva, DMGV. Pesquisa convergente assitencial: uso na elaboração de modelos de cuidado de enfermagem. Rev. Bras. Enferm. 2012 Nov/Dec;65(6). https://doi.org/10.1590/S003471672012000600019

10. Pivoto FL, Lunardi Filho WD, Santos SSC, Lunardi VL. Convergentassistential research: an integrative review of scientific nursing production. Texto contexto - enferm. [Internet]. 2013 Sep [cited 2017 Jan 30]; 22(3): 843-849. Available from: http://www.scielo.br/scielo.php?script=sci arttext\&pid=S0104-07072013000300034\&lng=en. http://dx.doi. org/10.1590/S0104-07072013000300034 [ Links ]

11. Etkind S, Bone AE, Lovell N, Cripps RL, Harding R, Higginson IJ et al. The role and response of palliative care and hospice services in epidemics 
and pandemics: a rapid review to inform practice during the COVID-19 pandemic. J Pain Symptom Manage. Articles In Press [Internet] 2020 [cited 2020 May 10]. Available from: 10.1016/j.jpainsymman.2020.03.029. 